\title{
Convergence and stability of stochastic parabolic functional differential equations
}

Zhao $\mathrm{Li}^{1}$ and Shuyong $\mathrm{Li}^{2 *}$

${ }^{\text {*Correspondence: }}$ shuyongli@263.net 2Mianyang Teachers' College, Mianyang, China

Full list of author information is available at the end of the article

\begin{abstract}
The main purpose of this paper is to investigate the convergence and stability of stochastic parabolic functional differential equations. Firstly, a comparison theorem in the context of Lyapunov-like function together with differential inequality is established. Secondly, various criteria for the convergence and stability are obtained on the basis of the comparison theorem and stochastic analysis techniques. Finally, two examples are provided to illustrate the significance of the theoretical results.
\end{abstract}

Keywords: Stochastic parabolic functional differential equations; Comparison theorem; Functional differential equation; Convergence; Stability

\section{Introduction}

In recent years, stochastic parabolic partial differential equations have received a great deal of attention due to their important applications in biological, control engineering, economics, physics, social sciences, and so on. Hence, the theory of stochastic parabolic partial differential equations has developed very quickly [1-3]. On the other hand, in many realistic models, their future states depend not only on the present states but also on the past ones. Therefore, time delay should be taken into consideration in modeling. As in [4], stochastic parabolic partial differential equations with time-delay are called stochastic parabolic functional differential equations (also called SPFDEs for short). The study of SPFDEs is very complex and difficult since most of them do not have an explicit solution. Thus, many researchers have been interested in investigating the stability; all kinds of ways to study stability of the solutions have been put out in [5-10] and the references cited therein. For example, Caraballo and Liu [5] have analyzed the exponential stability for mild solution to stochastic partial differential equations with delays by utilizing the well-known Gronwall inequality, and they imposed the requirement of the monotone decreasing behavior of the delays. Liu [6] has considered the exponential stability for SPFDEs by means of the Razumikhin-type theorem. Luo [7] has established some sufficient conditions ensuring the exponential stability and asymptotic stability for mild solution of stochastic partial differential equations with delays basing on the fixed point theorem. Taniguchi [8] has proved the almost sure exponential stability of mild solution for stochastic partial functional differential equations by using the analytic technique. Taniguchi [9] has investi- 
gated the exponential stability for stochastic delay partial differential equations by energy inequality. Wang and Yang [10] have given some sufficient conditions for determining the domain of $p$ th-moment stability region for stochastic partial differential equation with delays on the basis of the properties of nonnegative matrices, stochastic convolution, and the inequality technique.

It is well known that in previous work by Lakshmikatham, Leela and Ladde [11, 12], comparison principle as an important method has been successfully used in investigating the convergence and stability of differential equations. The main characteristic of this principle in stability analysis is that, by considering the relations which are based on a suitable Lyapunov-like function (or Lyapunov-like functional) together with the techniques of differential inequality between the original system and a comparison system, the stability of the solutions of the comparison system will imply the corresponding stability properties of the solutions of the original system. Usually, the stability of the comparison system (e.g., ordinary differential equations and linear differential difference equation) is obtained easily. Recently, Anabtawi and Sathananthan [13] employed the comparison principle and Lyapunov-like functional [14] techniques to study the convergence and stability behavior of stochastic parabolic partial differential equations.

Motivated by the above, we have the main aim in this paper to investigate the convergence and stability for SPFDEs. A comparison theorem in the context of Lyapunov-like function will be established. By the comparison theorem, some sufficient conditions for the convergence and stability of SPFDEs can be obtained by considering the convergence and stability of deterministic functional differential equation.

This paper is organized as follows. In Sect. 2, we introduce the basic notations and set up the comparison theorem for SPFDEs. In Sect. 3 and Sect. 4, we get our main results on the convergence and stability of solution process. In Sect. 5, we illustrate the significance of our results by two examples. Finally, conclusions are drawn in Sect. 6 .

\section{Preliminaries}

Let $\left(\Omega, \mathscr{F},\left\{\mathscr{F}_{t}\right\}_{t \geq t_{0}}, \mathbb{P}\right)$ be a complete probability space with a filtration $\left\{\mathscr{F}_{t}\right\}_{t \geq t_{0}}$ satisfying the usual conditions, $W(t)$ is an $m$-dimensional normalized Brownian motion defined on the complete probability space, and the increment $W(t+h)-W(t)$ is independent of every event in $\mathscr{F}_{t}$. Let $\mathbb{R}^{n}$ and $\mathbb{R}^{n \times m}$ denote the $n$-dimensional real Euclidean space and the set of all $n \times m$ real matrices, respectively. Let $H$ be a bounded domain in $\mathbb{R}^{d}$ with a smooth boundary $\partial H$. Let $\tau \geq 0$ be a bounded constant, $t_{0} \in \mathbb{R}_{+}=[0,+\infty), J=\left[t_{0},+\infty\right)$, $J_{\tau}=\left[t_{0}-\tau,+\infty\right), Q_{t_{0}}=J \times H$, and $\Gamma_{t_{0}}=J \times \partial H$. Let $C(X, Y)$ be the space of continuous mappings from the topological space $X$ to the topological space $Y$. Especially, let $C_{1} \triangleq C\left([-\tau, 0] \times H ; \mathbb{R}^{n}\right)$ denote the Banach space of all continuous functions $\varphi(s, x)$ from $[-\tau, 0] \times H$ to $\mathbb{R}^{n}$ with the norm $\|\varphi\|_{0}=\sup _{-\tau \leq s \leq 0}\|\varphi(s)\|$, where $\|\cdot\|$ is some norm in $H$; let $C_{2} \triangleq C\left([-\tau, 0] ; \mathbb{R}_{+}\right)$denote the family of all continuous functions $\phi$ from $[-\tau, 0]$ to $\mathbb{R}_{+}$ with the norm $|\phi|_{0}=\sup _{-\tau \leq s \leq 0} \phi(s)$. Denote by $L_{\mathscr{F}_{t}}^{p}\left(\Omega, C_{1}\right)$ the family of all $\mathscr{F}_{t}$ measurable, $C_{1}$-valued random variables $\varphi(s, x)$ with $\sup _{-\tau \leq s \leq 0} \mathbb{E}\|\varphi(s)\|^{p}<\infty$, where $\{\mathbb{E}(\cdot)\}$ stands for the mathematical expectation operator with respect to the given probability measure $\mathbb{P}$, the superscript " $T$ " denotes the transpose of a matrix or vector, $\operatorname{Tr}(\cdot)$ denotes the trace of the corresponding matrix. 
In the present paper, we consider the following SPFDEs with initial boundary value problem:

$$
\left\{\begin{array}{l}
d u=F\left(t, x, u(t, x), u_{t}(s, x), \frac{\partial u}{\partial x}, \frac{\partial^{2} u}{\partial x^{2}}\right) d t+G\left(t, x, u(t, x), u_{t}(s, x)\right) d W(t), \quad(t, x) \in Q_{t_{0}}, \\
u_{t_{0}}(s, x)=\varphi(s, x), \quad s \in[-\tau, 0], x \in H, \\
u(t, x)=\psi(t, x), \quad(t, x) \in \Gamma_{t_{0}},
\end{array}\right.
$$

where $u(t, x)=\left(u_{1}(t, x), u_{2}(t, x), \ldots, u_{n}(t, x)\right)^{T}, u_{t}(s, x)=u(t+s, x), s \in[-\tau, 0], x \in H$, $F \in C\left(Q_{t_{0}} \times \mathbb{R}^{n} \times C_{1} \times \mathbb{R}^{n d} \times \mathbb{R}^{n d^{2}} ; \mathbb{R}^{n}\right)$ is elliptic, $G \in C\left(Q_{t_{0}} \times \mathbb{R}^{n} \times C_{1} ; \mathbb{R}^{n \times m}\right), \frac{\partial u}{\partial x}=$ $\left(\frac{\partial u_{1}}{\partial x}, \frac{\partial u_{2}}{\partial x}, \ldots, \frac{\partial u_{n}}{\partial x}\right)^{T}, \frac{\partial u_{i}}{\partial x}=\left(\frac{\partial u_{i}(t, x)}{\partial x_{1}}, \frac{\partial u_{i}(t, x)}{\partial x_{2}}, \ldots, \frac{\partial u_{i}(t, x)}{\partial x_{d}}\right), \frac{\partial^{2} u}{\partial x^{2}}=\left(\frac{\partial^{2} u_{1}(t, x)}{\partial x^{2}}, \frac{\partial^{2} u_{2}(t, x)}{\partial x^{2}}, \frac{\partial^{2} u_{n}(t, x)}{\partial x^{2}}\right)^{T}, \frac{\partial^{2} u_{i}}{\partial x^{2}}=$ $\left(\frac{\partial^{2} u_{i}(t, x)}{\partial x_{1}^{2}}, \frac{\partial^{2} u_{i}(t, x)}{\partial x_{1} \partial x_{2}}, \ldots, \frac{\partial^{2} u_{i}(t, x)}{\partial x_{d}^{2}}\right)$.

In the paper, we always assume that $F, G$, and the domain are smooth enough so that Eq. (2.1) has a solution process on $J \times H$. Further, assume that $F(t, x, 0,0,0,0) \equiv 0$ and $G(t, x, 0,0) \equiv 0$. Then Eq. (2.1) obviously has a trivial solution $u(t, x) \equiv 0$.

Denote by $C^{1,2}\left(\mathbb{R} \times S_{\rho} ; \mathbb{R}_{+}\right)$the space of all nonnegative functions $V(t, u)$ defined on $\mathbb{R} \times S_{\rho}$ which are once continuously differential in $t$ and twice in $u$, where $S_{\rho} \equiv\{(t, x) \in$ $\mathbb{R} \times H:\|u(t, x)\|<\rho\}$.

Theorem 2.1 Let $V(t, u) \in C^{1,2}\left(J_{\tau} \times S_{\rho} ; \mathbb{R}_{+}\right), V(t, u)$ be Lipschitzian in $u$ for fixed $t$. Further assume that

(i) $g(t, y, \sigma) \in C\left(J \times \mathbb{R}_{+} \times C_{2} ; \mathbb{R}\right), g(t, y, \sigma)$ is concave and nondecreasing in $\sigma$ for each $(t, y)$ and satisfies

$$
\mathcal{L} V(t, u(t, x)) \leq g\left(t, V, V_{t}\right)
$$

where $V_{t}=V(t+s, u(t+s, x)), s \in[-\tau, 0], x \in H$, and

$$
\begin{aligned}
\mathcal{L} V(t, u(t, x)) \\
=\frac{\partial V(t, u(t, x))}{\partial t} \\
\quad+\frac{\partial V(t, u(t, x))}{\partial u} F\left(t, x, u(t, x), u_{t}(s, x), \frac{\partial u(t, x)}{\partial u}, \frac{\partial^{2} u(t, x)}{\partial u^{2}}\right) \\
\quad+\frac{1}{2} \operatorname{Tr}\left[\left(G\left(t, x, u(t, x), u_{t}(s, x)\right)\right)^{T} \frac{\partial^{2} V(t, u(t, x))}{\partial u^{2}} G\left(t, x, u(t, x), u_{t}(s, x)\right)\right],
\end{aligned}
$$

where $\frac{\partial V(t, u(t, x))}{\partial u}=\left(\frac{\partial V(t, u(t, x))}{\partial u_{1}}, \ldots, \frac{\partial V(t, u(t, x))}{\partial u_{n}}\right), \frac{\partial^{2} V(t, u(t, x))}{\partial u^{2}}=\left(\frac{\partial^{2} V(t, u(t, x))}{\partial u_{i} \partial u_{j}}\right)_{n \times n} ;$

(ii) $r\left(t ; t_{0}, \xi(s)\right)$ is the maximal solution of the functional differential equation

$$
\left\{\begin{array}{l}
\frac{d y}{d t}=g\left(t, y, y_{t}\right), \quad t \in J, \\
y_{t_{0}}=\xi(s) \in C_{2}, \quad s \in[-\tau, 0],
\end{array}\right.
$$

where $y_{t}=y(t+s)$; 
(iii) for the solution process $u(t, x)$ of SPFDEs (2.1), $\mathbb{E}[V(t, u(t, x))]$ exists for $t \in J_{\tau}$, and

$$
\begin{cases}\mathbb{E} V\left(t_{0}+s, u\left(t_{0}+s, x\right)\right) \leq \xi(s), & s \in[-\tau, 0], x \in H \\ \mathbb{E} V(t, \psi(t, x)) \leq r\left(t ; t_{0}, \xi(s)\right), & (t, x) \in \Gamma_{t_{0}} .\end{cases}
$$

These assumptions imply

$$
\mathbb{E} V(t, u(t, x)) \leq r\left(t ; t_{0}, \xi(s)\right), \quad t \in J
$$

Proof Let $u(t, x)$ be any solution of SPFDEs (2.1). Applying Itô's formula to $V(t, u(t, x))$, we have

$$
\begin{aligned}
d V(t, u(t, x))= & \mathcal{L} V(t, u(t, x)) d t \\
& +\frac{\partial V(t, u(t, x))}{\partial u} G\left(t, x, u(t, x), u_{t}(s, x)\right) d W(t) \quad \text { (a.s.), }
\end{aligned}
$$

where the operator $\mathcal{L}$ is defined as (2.3).

For small $h>0$,

$$
\begin{aligned}
v(t+h)-v(t) & =\mathbb{E} V(t+h, u(t+h, x))-\mathbb{E} V(t, u(t, x)) \\
& =\mathbb{E} \int_{t}^{t+h} \mathcal{L} V(\theta, u(\theta, x)) d \theta \leq \mathbb{E} \int_{t}^{t+h} g\left(\theta, V, V_{\theta}\right) d \theta \\
& \leq \int_{t}^{t+h} g\left(\theta, \mathbb{E} V, \mathbb{E} V_{\theta}\right) d \theta \quad \text { (by Jensen's inequality), }
\end{aligned}
$$

where $v(t)=\mathbb{E} V(t, u(t, x))$.

Dividing both sides by $h>0$ and taking the limit superior as $h \rightarrow 0^{+}$, we obtain

$$
D^{+} v(t)=\lim _{h \rightarrow 0^{+}} \sup \frac{1}{h}[v(t+h)-v(t)] \leq g\left(t, v(t), v_{t}\right),
$$

where $D^{+} v(t)$ is Dini's derivative of $v(t)$.

By Theorem 8.1.4 in [11], we have

$$
v(t)=\mathbb{E} V(t, u(t, x)) \leq r\left(t ; t_{0}, \xi(s)\right), \quad t \in J
$$

This completes the proof of Theorem 2.1 .

\section{Convergence analysis}

In this section, we utilize the comparison theorem in the context of Lyapunov-like function established in Sect. 2 to investigate the convergence of the solution process of SPFDEs (2.1). Let $u(t, x, \omega)$ be any solution process of SPFDEs (2.1).

Definition 3.1 A solution process $u(t, x, \omega)$ of $(2.1)$ is said to

(i) converge in the $p$ th moment if, for every $\varepsilon>0$ and $t_{0} \in J$, there exist $\delta>0$ and a positive number $T=T\left(\varepsilon, \delta, t_{0}\right)>0$ such that

$$
\mathbb{E}\left(\|u(t, \omega)\|^{p}\right)<\varepsilon, \quad \text { for } t \geq T+t_{0},
$$

whenever $\sup _{-\tau \leq s \leq 0} \mathbb{E}\|\varphi(s, \omega)\|^{p} \leq \delta$ and $\mathbb{E}\left(\|\psi(t, \omega)\|^{p}\right)<\varepsilon, t \geq T+t_{0}$; 
(ii) converge in probability if, for every $\varepsilon>0, \mu>0$, and $t_{0} \in J$, there exist $\delta>0$ and a positive number $T=T\left(t_{0}, \varepsilon, \delta, \mu\right)$ such that

$$
P\{\omega:\|u(t, \omega)\| \geq \varepsilon\}<\mu, \quad t \geq T+t_{0}
$$

whenever $\sup _{-\tau \leq s \leq 0} \mathbb{E}\|\varphi(s, \omega)\|^{p} \leq \delta$ and $P\{\omega:\|\psi(t, \omega)\| \geq \varepsilon\}<\mu, t \geq T+t_{0}$.

Definition 3.2 The maximal solution of functional differential equation (2.4) through $\left(t_{0}, \xi(s)\right)$ is said to converge if, for every $\varepsilon>0$ and $t_{0} \in J$, there exist $\delta>0$ and a positive number $T=T\left(\varepsilon, \delta, t_{0}\right)>0$ such that $|\xi|_{0} \leq \delta$ implies

$$
r\left(t ; t_{0}, \xi(s)\right)<\varepsilon, \quad \text { for } t \geq T+t_{0},
$$

where $\xi \in C_{2},|\xi|_{0}=\sup _{-\tau \leq s \leq 0} \xi(s)$.

\section{Theorem 3.3 Assume that}

(i) all the hypotheses of Theorem 2.1 hold;

(ii) $V(t, u)$ has the unique zero at $u \equiv 0$;

(iii) for $(t, u) \in J_{\tau} \times \mathbb{R}^{n}, V(t, u)$ satisfies the following inequality:

$$
b\left(\|u(t)\|^{p}\right) \leq V(t, u(t, x))
$$

where $b \in \mathcal{V K}, \mathcal{V K}$ is the collection of all continuous, convex, and increasing functions defined on $\mathbb{R}^{+}$into itself with $b(0)=0$ and $b(r) \rightarrow \infty$ as $r \rightarrow \infty$;

(iv) the maximal solution $r\left(t ; t_{0}, \xi(s)\right)$ of $(2.4)$ through $\left(t_{0}, \xi(s)\right)$ converges to zero as $t \rightarrow \infty$.

Then the solution process of SPFDEs (2.1) converges in the pth moment to the zero vector.

Proof Assume that the maximal solution of (2.4) is convergent. Let $\varepsilon>0$ and $t_{0} \in J$. Then there exist $\delta=\delta\left(t_{0}, \varepsilon\right)>0$ and $T=T\left(\varepsilon, \delta, t_{0}\right)$ such that when $|\xi|_{0} \leq b(\delta)$, we get

$$
r\left(t ; t_{0}, \xi(s)\right)<b(\varepsilon), \quad \text { for } t \geq T+t_{0} .
$$

Choose $\xi(s)$ such that $\mathbb{E} V\left(t_{0}+s, \varphi(s, x, \omega)\right) \leq \xi(s)$ and $\mathbb{E} V(t, \psi(t, x, \omega)) \leq r\left(t ; t_{0}, \xi(s)\right)$ on $\Gamma_{t_{0}}$. This together with (3.4) and (3.5) implies that

$$
\begin{aligned}
b\left[\mathbb{E}\left(\|\varphi(s, \omega)\|^{p}\right)\right] & \leq \mathbb{E}\left[b\left(\|\varphi(s, \omega)\|^{p}\right)\right] \\
& \leq \mathbb{E} V\left(t_{0}+s, \varphi(s, x, \omega)\right) \leq|\xi|_{0} \leq b(\delta), \quad-\tau \leq s \leq 0,
\end{aligned}
$$

and

$$
\begin{aligned}
b\left[\mathbb{E}\left(\|\psi(t, \omega)\|^{p}\right)\right] & \leq \mathbb{E}\left[b\left(\|\psi(t, \omega)\|^{p}\right)\right] \leq \mathbb{E} V(t, \psi(t, x, \omega)) \\
& \leq r\left(t ; t_{0}, \xi(s)\right)<b(\varepsilon), \quad t \geq T+t_{0} .
\end{aligned}
$$

By the property of $b$, it reduces to

$$
\sup _{-\tau \leq s \leq 0} \mathbb{E}\|\varphi(s, \omega)\|^{p} \leq \delta \quad \text { and } \quad \mathbb{E}\|\psi(t, \omega)\|^{p}<\varepsilon, \quad t \geq T+t_{0}
$$


From the conclusion of Theorem 2.1, we have $\mathbb{E} V(t, u(t, x, \omega))<r\left(t ; t_{0}, \xi(s)\right)$.

Now we claim that $\sup _{-\tau \leq s \leq 0} \mathbb{E}\|\varphi(s, \omega)\|^{p} \leq \delta$ and $\mathbb{E}\|\psi(t, \omega)\|^{p}<\varepsilon$ for $t \geq T+t_{0}$ imply $\mathbb{E}\left(\|u(t, \omega)\|^{p}\right)<\varepsilon, t \geq T+t_{0}$. Suppose it is not true, there exists a sequence $\left\{t_{j}\right\}$ such that $t_{j} \geq T+t_{0}, t_{j} \rightarrow \infty$ as $j \rightarrow \infty$, and $\mathbb{E}\left(\left\|u\left(t_{j}, \omega\right)\right\|^{p}\right)=\varepsilon$.

From (3.4), (3.5), the convexity of $b$, and Jensen's inequality, we have

$$
\begin{aligned}
b(\varepsilon) & =b\left(\mathbb{E}\left\|u\left(t_{j}, \omega\right)\right\|^{p}\right) \leq \mathbb{E} b\left(\left\|u\left(t_{j}, \omega\right)\right\|^{p}\right) \leq \mathbb{E} V\left(t_{j}, u\left(t_{j}, x, \omega\right)\right) \\
& \leq r\left(t_{j} ; t_{0}, \xi(s)\right)<b(\varepsilon),
\end{aligned}
$$

which is a contradiction. Therefore our claim is true, this completes the proof of Theorem 3.3.

\section{Theorem 3.4 Assume that}

(i) all the hypotheses of Theorem 2.1 hold;

(ii) $V(t, u)$ has the unique zero at $u \equiv 0$;

(iii) for $(t, u) \in J_{\tau} \times \mathbb{R}^{n}, V(t, u)$ satisfies the following inequality:

$$
b(\|u(t)\|) \leq V(t, u(t, x))
$$

(iv) the maximal solution $r\left(t ; t_{0}, \xi(s)\right)$ of (2.4) through $\left(t_{0}, \xi(s)\right)$ converges to zero as

$$
t \rightarrow \infty \text {. }
$$

Then the solution process of SPFDEs (2.1) converges in probability to the zero vector.

Proof Assume that the maximal solution of (2.4) is convergent. For giving $\mu>0$ and $\varepsilon>0$, we have $\mu b(\varepsilon)>0$. Then there exist $b(\delta)>0$ and $T=T\left(\varepsilon, \delta, t_{0}, \mu\right)$ such that

$$
r\left(t ; t_{0}, \xi(s)\right)<\mu b(\varepsilon), \quad \text { for } t \geq T+t_{0},
$$

whenever $|\xi|_{0}<b(\delta)$.

Choose $\xi(s)$ such that $\mathbb{E} V\left(t_{0}+s, \varphi(s, x, \omega)\right) \leq \xi(s)$ and $\mathbb{E} V(t, \psi(t, x, \omega)) \leq r\left(t ; t_{0}, \xi(s)\right)$. This together with (3.9) and (3.10) gives

$$
\begin{aligned}
& b(\mathbb{E}\|\varphi(s, \omega)\|) \leq \mathbb{E} b(\|\varphi(s, \omega)\|) \leq \mathbb{E} V\left(t_{0}+s, \varphi(s, x, \omega)\right) \leq|\xi|_{0}<b(\delta), \\
& \mathbb{E} b(\|\psi(t, \omega)\|) \leq \mathbb{E} V(t, \psi(t, x, \omega)) \leq r\left(t ; t_{0}, \xi(s)\right)<\mu b(\varepsilon) .
\end{aligned}
$$

From the property of $b$, it reduces to $\sup _{-\tau \leq s \leq 0} E\|\varphi(s, \omega)\|<\delta$. By (3.10) and (3.12), using Chebyshev's inequality, we reduce it to

$$
P\{\omega:\|\psi(t, \omega)\| \geq \varepsilon\} \leq \frac{\mathbb{E}(b\|\psi(t, \omega)\|)}{b(\varepsilon)}<\frac{\mu b(\varepsilon)}{b(\varepsilon)}=\mu, \quad t \geq T+t_{0} .
$$

Now we claim that $\sup _{-\tau \leq s \leq 0} E\|\varphi(s, \omega)\|<\delta$ and $P\{\omega:\|\psi(t, \omega)\| \geq \varepsilon\}<\mu$ for $t \geq T+t_{0}$ imply $P\{\omega:\|u(t, \omega)\| \geq \varepsilon\}<\mu, t \geq T+t_{0}$. Suppose it is not true, there exists a sequence $\left\{t_{j}\right\}$ such that $t_{j} \geq T+t_{0}, t_{j} \rightarrow \infty$ as $j \rightarrow \infty$, and $P\left\{\omega:\left\|u\left(t_{j}, \omega\right)\right\| \geq \varepsilon\right\} \geq \mu$.

By (3.9), (3.10), and Theorem 2.1, we have

$$
\mathbb{E} b\left(\left\|u\left(t_{j}, \omega\right)\right\|\right) \leq \mathbb{E} V\left(t_{j}, u\left(t_{j}, x, \omega\right)\right) \leq r\left(t_{j} ; t_{0}, \xi(s)\right)<\mu b(\varepsilon) .
$$


By (3.14), using Chebyshev's inequality, we arrive at the contradiction

$$
\begin{aligned}
\mu & \leq P\left\{\omega:\left\|u\left(t_{j}, \omega\right)\right\| \geq \varepsilon\right\} \leq P\left\{\omega: V\left(t_{j}, u\left(t_{j}, x, \omega\right)\right) \geq b(\varepsilon)\right\} \\
& \leq \frac{\mathbb{E} V\left(t_{j}, u\left(t_{j}, x, \omega\right)\right)}{b(\varepsilon)}<\frac{\mu b(\varepsilon)}{b(\varepsilon)}=\mu .
\end{aligned}
$$

Therefore our claim is true, this completes the proof of Theorem 3.4.

\section{Stability analysis}

In this section, we consider the stability of the trivial solution process of SPFDEs (2.1) by means of the comparison theorem.

Definition 4.1 The trivial solution of SPFDEs (2.1) is said to be:

(i) Stable in the $p$ th moment if, for each $\varepsilon>0$ and for all $t \in J$, there exists a constant $\delta=\delta\left(t_{0}, \varepsilon\right)>0$ such that

$$
\mathbb{E}\left(\|u(t, \omega)\|^{p}\right)<\varepsilon, \quad t \in J
$$

whenever $\sup _{-\tau \leq s \leq 0} \mathbb{E}\|\varphi(s, \omega)\|^{p} \leq \delta$ and $\mathbb{E}\left(\|\psi(t, \omega)\|^{p}\right)<\varepsilon$ for $t \in J$.

(ii) Asymptotically stable in the $p$ th moment if it is stable in the $p$ th moment, and if, for $\varepsilon>0, t_{0} \in J$, there exist positive numbers $\delta_{0}=\delta_{0}\left(t_{0}\right)$ and $T=T\left(t_{0}, \varepsilon\right)$ such that $\sup _{-\tau \leq s \leq 0} \mathbb{E}\|\varphi(s, \omega)\|^{p} \leq \delta$ and $\mathbb{E}\left(\|\psi(t, \omega)\|^{p}\right)<\varepsilon$ for $t \geq T+t_{0}$ imply $\mathbb{E}\left(\|u(t, \omega)\|^{p}\right)<\varepsilon, t \geq T+t_{0}$.

(iii) Stable in probability if, for each $\varepsilon>0, \mu>0, t_{0} \in J$, there exists a positive constant $\delta=\delta\left(t_{0}, \varepsilon, \mu\right)$ such that

$$
P\{\omega:\|u(t, \omega)\| \geq \varepsilon\}<\mu, \quad t \in J
$$

whenever $\sup _{-\tau \leq s \leq 0} \mathbb{E}\|\varphi(s, \omega)\|^{p} \leq \delta$ and $P\{\omega:\|\psi(t, \omega)\| \geq \varepsilon\}<\mu$.

(iv) Asymptotically stable in probability if it is stable in probability and, for any $\varepsilon>0$, $\mu>0, t_{0} \in J$, there exist numbers $\delta_{0}=\delta_{0}\left(t_{0}\right)$ and $T=T\left(t_{0}, \varepsilon, \mu\right)>0$ such that $\sup _{-\tau \leq s \leq 0} \mathbb{E}\|\varphi(s, \omega)\|^{p} \leq \delta$ and $P\{\omega:\|\psi(t, \omega)\| \geq \varepsilon\}<\mu, t \geq T+t_{0}$ imply $P\{\omega:\|u(t, \omega)\| \geq \varepsilon\}<\mu, t \geq T+t_{0}$.

Definition 4.2 The trivial solution $y=0$ of functional differential equation (2.4) through $\left(t_{0}, \xi(s)\right)$ is said to be:

(i) Stable if, for each $\varepsilon>0, t_{0} \in J$, there exists a positive constant $\delta\left(t_{0}, \varepsilon\right)$ such that when $|\xi|_{0} \leq \delta$ it implies

$$
y\left(t ; t_{0}, \xi(s)\right)<\varepsilon, \quad t \in J
$$

where $y\left(t ; t_{0}, \xi(s)\right)$ is the solution of Eq. (2.4) through $\left(t_{0}, \xi(s)\right)$.

(ii) Asymptotically stable if it is stable and converges to zero.

Theorem 4.3 In addition to the hypotheses of Theorem 3.3, assume that

$$
b\left(\|u(t)\|^{p}\right) \leq V(t, u(t, x)) \leq a\left(t,\|u(t)\|^{p}\right),
$$


where $a \in \mathcal{C K}, \mathcal{C K}$ is the collection of all continuous, concave, and increasing functions in $u$ for each $t \in \mathbb{R}_{+}$defined on $\mathbb{R}_{+} \times \mathbb{R}_{+}$into $\mathbb{R}_{+}$with $a(t, 0) \equiv 0$.

Then the trivial solution of (2.4) is stable implies the trivial solution of SPFDEs (2.1) is stable in the pth moment; the trivial solution of (2.4) is asymptotically stable implies the trivial solution of SPFDEs (2.1) is asymptotically stable in the pth moment.

Proof Assume that the trivial solution of (2.4) is stable. Let $\varepsilon>0$ and $t_{0} \in J$. Then there exists $\delta_{1}=\delta_{1}\left(t_{0}, \varepsilon\right)>0$ such that when $|\xi|_{0} \leq \delta_{1}$, we have $y\left(t ; t_{0}, \xi(s)\right)<b(\varepsilon), t \in J$. Hence, we get

$$
r\left(t ; t_{0}, \xi(s)\right)<b(\varepsilon), \quad t \in J
$$

Choose $\xi(s)$ such that $\mathbb{E} V\left(t_{0}+s, \varphi(s, x, \omega)\right) \leq \xi(s), \mathbb{E} V(t, \psi(t, x, \omega)) \leq r\left(t ; t_{0}, \xi(s)\right)$, and

$$
a\left(t_{0}+s, \mathbb{E}\left(\|\varphi(s, \omega)\|^{p}\right)\right)=\xi(s), \quad s \in[-\tau, 0] .
$$

Since $a \in \mathcal{C K}$, we can find a $\delta=\delta\left(t_{0}, \varepsilon\right)$ such that

$$
a\left(t_{0}+s, \mathbb{E}\left(\|\varphi(s, \omega)\|^{p}\right)\right)=\xi(s)<\delta_{1} \quad \text { and } \quad \sup _{-\tau \leq s \leq 0} \mathbb{E}\|\varphi(s, \omega)\|^{p} \leq \delta
$$

hold simultaneously.

From (4.4) and (4.5), we have

$$
\begin{aligned}
b\left(\mathbb{E}\|\psi(t, \omega)\|^{p}\right) & \leq \mathbb{E} b\left(\|\psi(t, \omega)\|^{p}\right) \leq \mathbb{E} V(t, \psi(t, x, \omega)) \\
& \leq r\left(t ; t_{0}, \xi(s)\right)<b(\varepsilon) .
\end{aligned}
$$

From (4.7) and (4.8), using the convexity of $b$, we have

$$
\sup _{-\tau \leq s \leq 0} \mathbb{E}\|\varphi(s, \omega)\|^{p} \leq \delta \quad \text { and } \quad \mathbb{E}\|\psi(t, \omega)\|^{p}<\varepsilon
$$

By Theorem 2.1, we have

$$
b\left(\mathbb{E}\|u(t, \omega)\|^{p}\right)<b(\varepsilon), \quad t \in J .
$$

So, we have $\mathbb{E}\|u(t, \omega)\|^{p}<\varepsilon, t \in J$.

Similar to the proof of Theorem 3.3, we can prove that the trivial solution of (2.4) is asymptotically stable implies the trivial solution of SPFDEs (2.1) is asymptotically stable in the $p$ th moment. The details are omitted.

This completes the proof of Theorem 4.3.

Theorem 4.4 In addition to the hypotheses of Theorem 3.4, assume that

$$
b(\|u(t)\|) \leq V(t, u(t, x)) \leq a(t,\|u(t)\|) .
$$

Then the trivial solution of (2.4) is stable implies the trivial solution of SPFDEs (2.1) is stable in probability; the trivial solution of (2.4) is asymptotically stable implies the trivial solution of SPFDEs (2.1) is asymptotically stable in probability. 
Proof Assume that the trivial solution of (2.4) is stable. For giving $\varepsilon>0$ and $\mu>0$, there exists $\delta_{2}=\delta_{2}\left(t_{0}, \varepsilon, \mu\right)>0$ such that $y\left(t ; t_{0}, \xi(s)\right)<\mu b(\varepsilon), t \in J$ for $|\xi|_{0} \leq \delta_{2}$. So, we have

$$
r\left(t ; t_{0}, \xi(s)\right)<\mu b(\varepsilon), \quad t \in J .
$$

As $a(t, u) \in \mathcal{C K}$, choose $\xi(s)\left(0<|\xi|_{0}<\delta_{2}\right)$ and $\delta=\delta\left(t_{0}, \varepsilon, \mu\right)$ such that

$$
\sup _{-\tau \leq s \leq 0} \mathbb{E}\|\varphi(s, \omega)\| \leq \delta, \quad a\left(t_{0}+s, \mathbb{E}\|\varphi(s, \omega)\|\right) \leq \xi(s)
$$

and

$$
\mathbb{E} b(\|\psi(t, \omega)\|) \leq \mathbb{E} V(t, \psi(t, x, \omega)) \leq r\left(t ; t_{0}, \xi(s)\right)<\mu b(\varepsilon) .
$$

From (4.9) and (4.11), we obtain

$$
\mathbb{E} V\left(t_{0}+s, \varphi(s, x, \omega)\right) \leq \mathbb{E} a\left(t_{0}+s,\|\varphi(s, \omega)\|\right) \leq a\left(t_{0}+s, \mathbb{E}\|\varphi(s, \omega)\|\right) \leq \xi(s) .
$$

By (4.12) and Chebyshev's inequality, we have

$$
\begin{aligned}
P\{\omega:\|\psi(t, \omega)\| \geq \varepsilon\} & =P\{\omega: V(t, \psi(t, x, \omega)) \geq b(\varepsilon)\} \\
& \leq \frac{\mathbb{E} V(t, \psi(t, x, \omega))}{b(\varepsilon)}<\frac{\mu b(\varepsilon)}{b(\varepsilon)}=\mu .
\end{aligned}
$$

From (4.9) and (4.10), Theorem 2.1 and the above, we have

$$
\mathbb{E} b(\|u(t, \omega)\|) \leq \mathbb{E} V(t, u(t, x, \omega)) \leq r\left(t ; t_{0}, \xi(s)\right)<\mu b(\varepsilon) .
$$

Thus, we claim that the trivial solution of (2.1) is stable in probability

$$
P\{\omega:\|u(t, \omega)\| \geq \varepsilon\}<\mu, \quad t \in J .
$$

If it is not true, there would exist $t_{1}>t_{0}$ and $s \in[-\tau, 0]$ such that $t_{1}+s>t_{0}$ and

$$
P\left\{\omega:\left\|u\left(t_{1}, \omega\right)\right\| \geq \varepsilon\right\} \geq \mu .
$$

By (4.9), (4.15), and the proof of Theorem 2.1, we get

$$
\mathbb{E} b\left(\left\|u\left(t_{1}+s, \omega\right)\right\|\right) \leq \mathbb{E} V\left(t_{1}, u\left(t_{1}+s, x, \omega\right)\right) \leq r\left(t_{1} ; t_{0}, \xi(s)\right)<\mu b(\varepsilon) .
$$

By (4.16), using Chebyshev's inequality, we arrive at the contradiction

$$
\begin{aligned}
\mu & \leq P\left\{\omega:\left\|u\left(t_{1}+s, \omega\right)\right\| \geq \varepsilon\right\} \leq P\left\{\omega: V\left(t_{1}+s, u\left(t_{1}+s, x, \omega\right)\right) \geq b(\varepsilon)\right\} \\
& \leq \frac{\mathbb{E} V\left(t_{1}+s, V\left(t_{1}+s, u\left(t_{1}+s, x, \omega\right)\right)\right)}{b(\varepsilon)} \\
& <\frac{\mu b(\varepsilon)}{b(\varepsilon)}=\mu .
\end{aligned}
$$


Therefore our claim is true. Similar to the proof of Theorem 3.4, we also prove that the trivial solution of (2.4) is asymptotically stable implies the trivial solution of SPFDEs (2.1) is asymptotically stable in probability.

This completes the proof of Theorem 4.4.

\section{Example}

In this section we present two examples in order to illustrate our theory. Our aim is to examine the asymptotic stability of one-dimensional SPFDEs and two-dimensional SPFDEs.

Example 5.1 Consider the following one-dimensional SPFDEs:

$$
\left\{\begin{array}{l}
d u=\left[\alpha \frac{\partial^{2} u(t, x)}{\partial x^{2}}+\beta \frac{\partial u(t, x)}{\partial x}+\gamma u(t-\tau, x)-u^{3}(t, x)\right] d t+\varsigma u(t-\tau, x) d W(t), \\
\quad t \geq 0, x \in[0, \pi], \\
u\left(t_{0}+s, x\right)=\eta(s, x), \quad-\tau \leq s \leq 0, \\
u(t, 0)=u(t, \pi)=0,
\end{array}\right.
$$

where $u(t, x) \in \mathbb{R}_{+}, x \in[0, \pi], W(t)$ is a one-dimensional standard Brownian motion. $\alpha, \beta$, $\gamma$, and $\varsigma$ are positive numbers.

Let the Lyapunov function be as follows:

$$
V(t, u(t, x))=\frac{1}{2}\|u(t, x)\|^{2}
$$

where $\|u(t, x)\|=\left[\int_{0}^{\pi} u^{2}(t, x) d x\right]^{\frac{1}{2}}$.

Applying Itô's formula for $V(t, u(t, x))$, taking expectation, using Poincarés inequality together with Green's formulas and Schwarz inequality, we obtain

$$
\begin{aligned}
\mathcal{L} V(t, u(t, x)) \leq & \int_{0}^{\pi} u(t, x)\left[\alpha \frac{\partial^{2} u(t, x)}{\partial x^{2}}+\beta \frac{\partial u(t, x)}{\partial x}+\gamma u(t-\tau, x)-u^{3}(t, x)\right] d x \\
& +\frac{1}{2} \int_{0}^{\pi}(\varsigma u(t-\tau, x))^{2} d x \\
\leq & -\left(4 \pi^{2} \alpha-\gamma\right) V(t, u(t, x))+\left(\varsigma^{2}+\gamma\right) V(t-\tau, u(t-\tau, x)) .
\end{aligned}
$$

Our comparison system is

$$
\left\{\begin{array}{l}
\frac{d y}{d t}=g(t, y, y(t-\tau))=-\left(4 \pi^{2} \alpha-\gamma\right) y(t)+\left(\varsigma^{2}+\gamma\right) y(t-\tau) \\
y_{t_{0}}=\xi(s) \in C_{2}
\end{array}\right.
$$

So all the hypotheses of Theorem 4.3 are satisfied. From [15], we know that the trivial solution of system (5.4) is asymptotically stable when $\varsigma^{2}+2 \gamma<4 \pi^{2} \alpha$. By Theorem 4.3 , the solution process of SPFDEs (5.1) is asymptotically stable in the mean square in $L^{2}$ when $\varsigma^{2}+2 \gamma<4 \pi^{2} \alpha$ 
Example 5.2 Consider the following two-dimensional SPFDEs:

$$
\left\{\begin{aligned}
& d u_{i}(t, x)= {\left[\Delta u_{i}(t, x)+a_{i}(t) u_{i}(t, x)+\sum_{j=1}^{2} b_{i j}(t) u_{j}(t-\tau, x)\right] d t } \\
&+\sigma_{i}(t) u_{i}(t-\tau, x) d W_{i}(t), \quad(t, x) \in Q_{t_{0}}, i=1,2, \\
& u\left(t_{0}+s, x\right)= \varphi(s, x), \quad-\tau \leq s \leq 0, x \in H \\
& u(t, x)=0, \quad(t, x) \in \Gamma_{t_{0}}
\end{aligned}\right.
$$

where $a_{i}(t), b_{i j}(t), \sigma_{i}(t)(i, j=1,2)$ are continuous and bounded for $t \in J . b_{i j}(t) \geq 0, i, j=$ 1,2. $\Delta$ is a Laplacian operator, $u_{1}(t, x), u_{2}(t, x) \in \mathbb{R}_{+}, H$ is a bounded domain in $\mathbb{R}^{d}$ with a smooth boundary $\partial H$.

Let the Lyapunov function be as follows:

$$
V(t, u(t, x))=\|u(t, x)\|^{2},
$$

where $\|u(t, x)\|=\left[\int_{H}\left(u_{1}^{2}(t, x)+u_{2}^{2}(t, x)\right) d x\right]^{\frac{1}{2}}$.

Applying Itô's formula for $V(t, u(t, x))$, using Poincaré's inequality together with Green's formulas and Schwarz inequality, we obtain

$$
\begin{aligned}
\mathcal{L} V(t, u(t, x)) \\
=2 \int_{H} u_{1}(t, x)\left[\Delta u_{1}(t, x)+a_{1}(t) u_{1}(t, x)+b_{11}(t) u_{1}(t-\tau, x)\right. \\
\left.\quad+b_{12}(t) u_{2}(t-\tau, x)\right] d x+\sigma_{1}^{2}(t) \int_{H} u_{2}^{2}(t-\tau, x) d x+2 \int_{H} u_{2}(t, x)\left[\Delta u_{2}(t, x)\right. \\
\left.\quad+a_{2}(t) u_{2}(t, x)+b_{21}(t) u_{1}(t-\tau, x)+b_{22}(t) u_{2}(t-\tau, x)\right] d x \\
\quad+\sigma_{2}^{2}(t) \int_{H} u_{1}^{2}(t-\tau, x) d x \\
\leq\left(-\frac{2}{M}+\theta(t)\right) V(t, u(t, x))+\rho(t) V(t-\tau, u(t-\tau, x)),
\end{aligned}
$$

where $\theta(t)=\max \left\{2 a_{1}(t)+b_{11}(t)+b_{12}(t), 2 a_{22}(t)+b_{21}(t)+b_{22}(t)\right\}, \rho(t)=\max \left\{b_{11}(t)+b_{21}(t)+\right.$ $\left.\sigma_{1}^{2}(t), b_{12}(t)+b_{22}(t)+\sigma_{2}^{2}(t)\right\}, V(t, u(t, x))=\int_{H}\left[u_{1}^{2}(t, x)+u_{2}^{2}(t, x)\right] d x$, and $M$ depends on the domain of integration.

Our comparison system is

$$
\frac{d y}{d t}=g(t, y, y(t-\tau))=-\left(\frac{2}{M}-\theta(t)\right) y(t)+\rho(t) y(t-\tau) .
$$

From [15], system (5.8) is asymptotically stable if $\rho(t)+\theta(t)<\frac{2}{M}$.

It is obvious that all the hypotheses of Theorem 4.3 are satisfied. Therefore, the solution process of SPFDEs (5.5) is asymptotically stable in the mean square in $L^{2}$ if $\rho(t)+\theta(t)<\frac{2}{M}$.

\section{Conclusion}

In this paper, some sufficient conditions for the convergence and stability of the solution process of SPFDEs are established by developing the comparison principle in the context 
of Lyapunov-like function together with differential inequality. We believe that our approach and results are very efficient and comparable to the existing research in this area for stochastic parabolic partial differential equations [13]. We plan to extend this research result into stochastic parabolic functional differential equations with Markovian switching.

\section{Acknowledgements}

The authors would like to thank the reviewers for their constructive suggestions and helpful comments.

Funding

The research was supported by the National Natural Science Foundation of China (grant number 11571245).

\section{Competing interests}

The authors declare that there is no conflict of interest regarding the publication of this paper. The authors declare that they have no competing interests.

\section{Authors' contributions}

All authors read and approved the final manuscript.

\section{Author details}

${ }^{1}$ College of Mathematics and Software Science, Sichuan Normal University, Chengdu, China. ${ }^{2}$ Mianyang Teachers' College, Mianyang, China.

\section{Publisher's Note}

Springer Nature remains neutral with regard to jurisdictional claims in published maps and institutional affiliations.

Received: 15 April 2018 Accepted: 16 June 2018 Published online: 09 August 2018

\section{References}

1. Chow, P.L.: Stochastic Partial Differential Equations. Chapman \& Hall/CRC, Boca Raton (2015)

2. Wei, L., Zhu, Q.X., Duan, M.J., Yao, Z.S.: pth moment exponential stability of hybrid stochastic fourth-order parabolic equations. Adv. Differ. Equ. 2016, 65 (2016)

3. Anabtawi, M.J., Sathananthan, S.: Stability and convergence results for Itô-type parabolic partial differential equations in Hilbert spaces. Stoch. Anal. Appl. 27(4), 671-693 (2009)

4. Wang, C.Q:: A class of impulsive stochastic parabolic functional differential equations and their asymptotics. Acta Appl. Math. 146(1), 163-186 (2016)

5. Caraballo, T., Liu, K.: Exponential stability of mild solutions of stochastic partial differential equations with delays. Stoch. Anal. Appl. 17(5), 743-763 (1999)

6. Liu, K.: Stability of Infinite Dimensional Stochastic Differential Equations with Applications. Chapman \& Hall/CRC, Boca Raton (2006)

7. Luo, J.W.: Stability of stochastic partial differential equations with infinite delays. J. Comput. Appl. Math. 222(2), 364-371 (2008)

8. Taniguchi, T.: Almost sure exponential stability for stochastic partial functional differential equations. Stoch. Anal. Appl. 16(5), 965-975 (1998)

9. Taniguchi, T.: The exponential stability for stochastic delay partial differential equations. J. Math. Anal. Appl. 331(1), $191-205(2007)$

10. Wang, X.H., Yang, Z.G.: The domain of attraction and the stability region for stochastic partial differential equations with delays. Nonlinear Anal., Theory Methods Appl. 75(18), 6465-6472 (2012)

11. Lakshmikatham, V., Leela, S.: Differential and Integral Inequalities, vol. 2. Academic Press, New York (1969)

12. Ladde, G.S., Lakshmikatham, V.: Random Differential Inequalities. Academic Press, New York (1980)

13. Anabtawi, M.J., Sathananthan, S.: Stability and convergence via Lyapunov-like functionals of stochastic parabolic partial differential equations. Appl. Math. Comput. 157(1), 201-218 (2004)

14. Anabtawi, M.J.: Lyapunov-like functionals via Lyapunov functions for various types of stability of hybrid stochastic differential equation. J. Franklin Inst. 348(7), 1738-1750 (2001)

15. Hale, J.K.: Theory of Functional Differential Equations. Springer, New York (2003) 